\title{
Regulation and mechanisms of macrophage cholesterol efflux
}

\author{
Alan R. Tall, Philippe Costet, and Nan Wang \\ Division of Molecular Medicine, Department of Medicine, Columbia University, New York, New York, USA \\ J. Clin. Invest. 110:899-904 (2002). doi:10.1172/JCI200216391.
}

The accumulation of cholesterol in macrophage foam cells results from the uptake of retained and modified apoB lipoproteins (1) and is a central event in atherogenesis. Although it has long been known that HDL and its apolipoproteins can stimulate cholesterol efflux from macrophage foam cells $(2,3)$ and reduce atherogenesis in animal models $(4,5)$, this area of research has been enlivened by the elucidation of the genetic defect in Tangier disease, a condition in which there is a defect in apolipoprotein-mediated cholesterol efflux (6) and in which the hallmark pathology is the accumulation of macrophage foam cells in various tissues. Tangier disease is caused by mutations in the ATP-binding cassette transporter A1 (ABCA1). This cell surface transporter facilitates the efflux of phospholipids and cholesterol onto lipid-poor apolipoproteins, initiating the formation of HDL particles.

This Perspective will focus on recent studies on the regulation and role of ABCA1 in cellular cholesterol efflux, lipoprotein physiology, and atherogenesis, attempting to place this work in the broader context of studies on cellular cholesterol efflux, centripetal cholesterol transport, and $\mathrm{ABC}$ transporter structure and function.

\section{Expression and regulation of $A B C A 1$}

The ABCA1 mRNA is widely expressed and is found in the liver, kidney, adrenal gland, intestine, and CNS (7). It is also prominent in the macrophage foam cells of atherosclerotic lesions. While basal levels of ABCA1 mRNA and protein in macrophages are low, both are induced with cholesterol loading and can be reversed by HDL-mediated cholesterol efflux (8). The mechanism of cholesterol induction involves oxysteroldependent activation of the LXR/RXR transcription factors, which act on a DR4 site (direct repeat nuclear receptor-binding site with a spacing of four nucleotides) in the proximal promoter of the $A B C A 1$ gene.

\footnotetext{
Address correspondence to: Alan R. Tall, Department of Medicine, Division of Molecular Medicine, Columbia University, 630 West 168th Street 8-101, New York, New York 10032, USA. Phone: (212) 305-9418; Fax: (212) 305-5052;

E-mail: art1@columbia.edu.

Conflict of interest: No conflict of interest has been declared. Nonstandard abbreviations used: ATP-binding cassette (ABC); ATP-binding cassette transporter A1 (ABCA1); cerebrotendinous xanthomatosis (CTX); scavenger receptor B-I (SR-BI); knockout (KO).
}

$A B C A 1$ is one of a battery of LXR/RXR target genes involved in the regulation of centripetal cholesterol transport, cholesterol excretion, and fatty acid biosynthesis (Figure 1). Thus, LXR/RXR transcription factors regulate genes mediating macrophage cholesterol efflux ( $A P O E, A B C A 1$, and possibly $A B C G 1$ ), transport ( $L P L, C E T P$, and several genes encoding isoforms of apoC), conversion of cholesterol into bile acids (CYP7A), and metabolism and excretion into bile or intestinal lumen (ABCG5 and ABCG8). Small-molecule activators of LXR/RXR might therefore have a favorable effect on cholesterol homeostasis.

Since LXR is activated by specific oxysterols but apparently not by cholesterol itself, the induction of ABCA1 expression following cellular uptake of cholesterol-rich lipoproteins requires the conversion of cholesterol into specific oxysterols, such as $22-\mathrm{OH}$ cholesterol, $27-\mathrm{OH}$ cholesterol, or 24(S),25-epoxycholesterol. Cyp27, a mitochondrial enzyme that converts cholesterol into $27-\mathrm{OH}$ cholesterol, is mutated in cerebrotendinous xanthomatosis (CTX), and CTX fibroblasts fail to upregulate ABCA1 in response to cholesterol loading (ref. 9; for more discussion of the potential significance of oxysterols in cholesterol homeostasis, see Björkhem, this Perspective series, ref. 10). Since CTX patients develop xanthomatosis and premature atherosclerosis, macrophage foam cell formation in CTX may partly reflect a defect in ABCA1-mediated cholesterol efflux.

In addition to positive sterol regulation, $\mathrm{ABCA} 1$ is subject to negative regulation by non-sterol mevalonate products. Thus, geranylgeranylpyrophosphate inhibits $A B C A 1$ gene expression by different mechanisms, acting through the DR4 element in the $A B C A 1$ promoter (11). cAMP induces the ABCA1 mRNA in macrophages and some other cells by an unknown mechanism. A phosphodiesterase 4 inhibitor has been found to increase ABCA1 mRNA and cellular cholesterol efflux, suggesting a possible therapeutic approach to increasing ABCA1 expression (12). IFN- $\gamma$, conversely, decreases ABCA1 mRNA levels in cholesterol-loaded macrophages (13). Since IFN- $\gamma$ is proatherogenic, this finding provides a potential link between the inflammatory aspect of atherosclerosis and a defect in foam cell cholesterol efflux.

Early reports suggested that PPAR $\gamma$ activators increase $\mathrm{ABCA} 1$ transcription and cholesterol efflux by sequentially upregulating $\mathrm{LXR} \alpha$ and ABCA1. These 


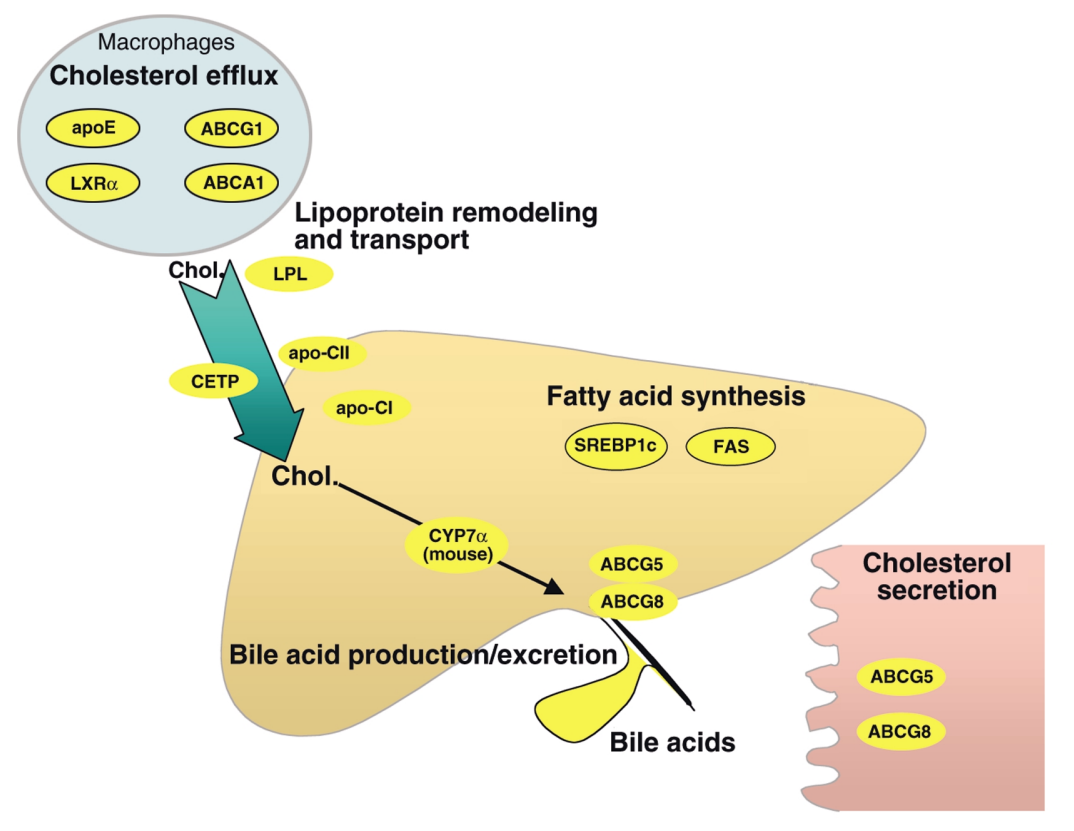

\begin{abstract}
Figure 1
LXR target genes and their products. LXR regulates cholesterol (Chol) efflux, centripetal cholesterol transport, cholesterol excretion, and fatty acid biosynthesis by inducing multiple genes involved in lipid metabolism. Two products of these genes, $A B C A 1$ and apoE, promote macrophage cholesterol efflux. Another LXR target, macrophage LPL, is activated by apoC-II, which is also regulated by LXR. Cholesteryl ester transfer protein (CETP) promotes transfer of HDL cholesteryl ester to VLDLs, which are taken up by the liver. In the liver, Cyp7 $\alpha$ (cytochrome $\mathrm{P} 4507 \alpha$ ) promotes cholesterol excretion by mediating conversion of cholesterol into bile acids. ABCG5 and ABCG8 facilitate hepatic and intestinal cholesterol excretion. LXR activates SREBP1c, regulating the expression of multiple lipogenic proteins, including FAS (fatty acid synthesis), which is also a direct target of LXR.
\end{abstract}

findings have been widely cited as an explanation for antiatherogenic effects of PPAR $\gamma$ activators. However, although the $L X R \alpha$ gene contains an authentic PPAR $\gamma$ response element (14), some recent reports have failed to confirm that PPAR $\gamma$ activators, such as troglitazone or rosiglitazone, induce ABCA1 mRNA and cholesterol efflux in macrophages $(15,16)$. Perhaps the availability of LXR/RXR ligands, rather than the abundance of LXRs themselves, is usually rate-limiting for ABCA1 expression in differentiated cells. Nevertheless, PPAR $\delta$ activators appear to induce ABCA1 expression and cholesterol efflux moderately, and they can increase HDL levels in an obese-monkey model (17).

There is also emerging evidence that ABCA1 is regulated at a posttranscriptional level: in addition to acting as inhibitors of LXR-stimulated gene expression (18), polyunsaturated fatty acids increase the already rapid turnover of ABCA1 in macrophages and can diminish cell surface expression of this protein (19). Since FFA levels may be increased in diabetes, this response suggests a mechanism that could produce a defect in cellular cholesterol efflux in poorly controlled diabetes. Lipid-poor apolipoproteins, conversely, can stabilize ABCA1 protein (20).

\section{Pathways mediating cellular cholesterol efflux}

Three distinct pathways of cellular cholesterol efflux involving HDL and its apolipoproteins have been described. First, plasma HDL particles can promote cholesterol efflux by a process of passive aqueous diffusion (21). Free cholesterol molecules spontaneously desorb from the plasma membrane, diffuse through the aqueous phase, and incorporate into HDL particles by collision. Second, scavenger receptor B-I (SR-BI), an HDL receptor that mediates the selective uptake of HDL cholesteryl esters into cells (22), also facilitates the efflux of cholesterol from cells to HDLs (23). Cholesterol efflux is blocked by antibodies that inhibit binding of HDLs to
SR-BI (24), suggesting that the mechanism involves a direct interaction between HDL particles and the receptor and is therefore distinct from the aqueous diffusion mechanism. Finally, ABCA1 mediates the active efflux of phospholipid and cholesterol from cells to lipid-poor apolipoproteins, such as apoA-I. ApoA-I binds and crosslinks to $A B C A 1$, suggesting a direct interaction that leads to lipid efflux (25).

Passive aqueous diffusion and SR-BI-facilitated efflux involve bidirectional exchange of cholesterol between cells and HDLs. In contrast, ABCA1/apolipoprotein-mediated cholesterol and phospholipid efflux is a unidirectional net transfer process (25). The specificity for cholesterol acceptors in the three pathways is different. Passive efflux is driven by the phospholipid content of lipoprotein acceptors (26). SR-BI can bind both apolipoproteins and HDL particles, but binding affinity is greatest for large, spherical HDL particles, suggesting that these may be the major substrate for SR-BI in vivo (27). In contrast, ABCA1 binds and cross-links lipid-poor apoA-I, while showing minor interaction with smaller $\mathrm{HDL}_{3}$ and no interaction with larger $\mathrm{HDL}_{2}$ subspecies (28). Thus, ABCA1 shows limited interaction with the major HDL species isolated from plasma, emphasizing the importance of a small pool of lipid-poor apolipoproteins either secreted by cells or generated by lipid exchange and lipolysis of HDL particles in the bloodstream. ABCA1 probably mediates the rapid efflux of cellular cholesterol in response to pre- $\beta$ HDL, a minor but metabolically significant fraction of plasma HDLs comprising free apoA-I and apoA-I associated with a small number of phospholipid and cholesterol molecules (29). Following addition of lipid to apoA-I by ABCA1, there is further growth of HDL particles as a result of phospholipid transfer by phospholipid transfer protein, cholesterol esterification by LCAT, and acquisition of apoE molecules. The large, apoE-rich HDLs are proba- 


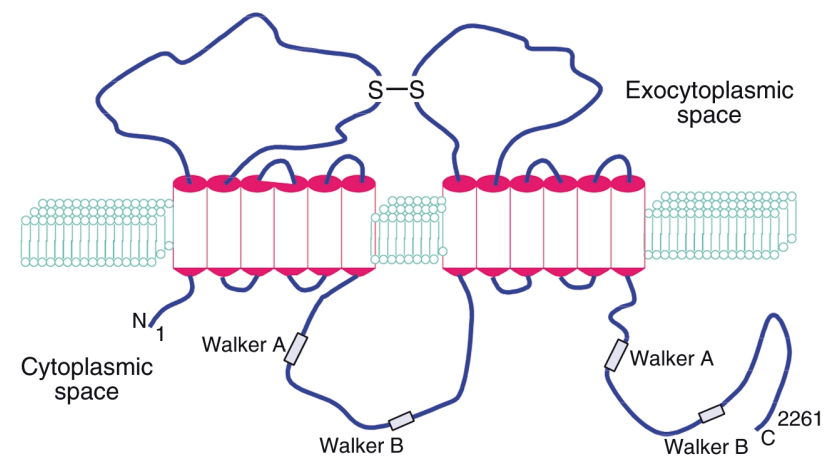

Figure 2

Schematic model of ABCA1. The model is based on studies by Bungert et al. (30) on ABCA4 membrane topology and by Fitzgerald et al. $(33,49)$ on $A B C A 1$ membrane topology. ABCA4 is the closest relative of $A B C A 1$.

bly the optimal substrates for SR-BI. In summary, ABCA1 may play the major role in initiating cholesterol efflux from macrophages and other cells to lipid-poor apolipoproteins, while SR-BI principally clears cholesterol and cholesteryl ester from large HDLs in the liver and steroidogenic tissues.

\section{ABC transporter-mediated lipid efflux}

$A B C$ transporter proteins comprise a large family of membrane proteins involved in the cellular export or import of a wide variety of different substances, including ions, lipids, cyclic nucleotides, peptides, and proteins. These transporters have been widely implicated in disease processes, such as Stargardt macular degeneration, cholestasis of pregnancy, and cystic fibrosis. In general, $\mathrm{ABC}$ transporters consist of two membranespanning domains forming a translocation pathway for a particular substrate, and two attached, cytoplasmic $\mathrm{ABCs}$ that link the transport process to the hydrolysis of ATP. ABCA1 conforms to this general model, and its likely membrane topology is shown in Figure 2. Similar to its closest neighbor (ABCR or ABCA4, the defective molecule in Stargardt retinal degeneration) (30), ABCA1 is likely to comprise two sets of six transmembrane domains, with two large extracellular loops joined by a disulfide bridge, and to have two paired cytoplasmic Walker and $A B C$ signature motifs (Figure 2).

In the presence of apoA-I, ABCA1 promotes cellular efflux of phosphatidylcholine, sphingomyelin, and cholesterol. Because ABCA1 interacts with different lipid-poor apolipoproteins, including apoA-I, apoA-II, apoE, and likely apoA-IV, it appears to recognize a general feature in the apolipoprotein structure, such as a cluster of amphipathic helices (31). ABCA1 cross-links to apoA-I $(25,32)$, suggesting a direct interaction, likely involving the first and fourth extracellular loop (33) (Figure 2), as well as other regions of the molecule. Mutation of the cytoplasmic ATP-binding Walker motif results in defective lipid efflux and abolishes binding and cross-linking of apoA-I (28). One interpretation is that apoA-I binding to ABCA1 is linked to binding of ATP to cytoplasmic domains and a conformational change that fosters translocation of phos- pholipid and access to apoA-I (28). Mutation of the Walker motif would prevent the formation of this transition state causing defective phospholipid translocation and binding of apoA-I. Another interpretation is that the binding site consists primarily of phospholipids translocated by ABCA1 and lipid efflux is not dependent on a direct protein-protein interaction between apoA-I and ABCA1 (34). A recent study using mutagenesis analysis of several Tangier disease missense mutations in the extracellular loop of ABCA1 showed that binding of apoA-I is necessary but not sufficient for lipid efflux (33). The identification of a mutant with increased cross-linking of apoA-I but defective lipid efflux provides strong evidence that the binding site does not consist of translocated lipid (33).

Lipid rafts and ABCA1-mediated cholesterol efflux Although the primary action of ABCA1 may be to act as a phospholipid translocase, different views have emerged on how ABCA1 mediates cholesterol efflux. One view is that ABCA1 promotes assembly of a cholesterol/phospholipid/apoA-I complex in a single step (Figure 3a). A variant of this model is that ABCA1 translocates phospholipid and cholesterol, giving rise to an excess of these lipids in the external membrane hemileaflet; apoA-I binds ABCA1 and absorbs the excess lipid, forming an HDL particle. A second view is that ABCA1 acts in a two-step process, initially giving rise to a phospholipid/apoA-I particle that then promotes cholesterol efflux from distinct cholesterolenriched membrane microdomains, such as cholesterol- and sphingolipid-rich rafts or caveolae (28) (Figure 3c). A third model, blending features of each of the two earlier models, holds that ABCA1 occupies a

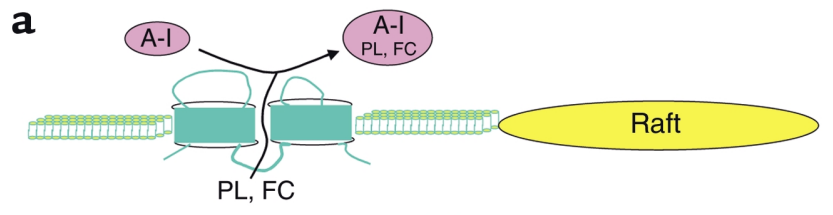

b

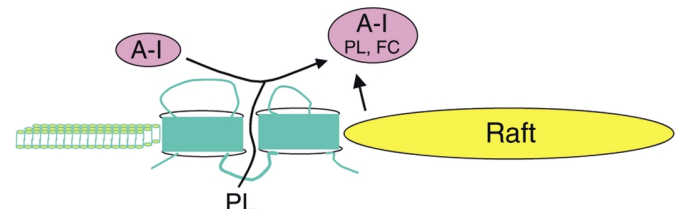

C

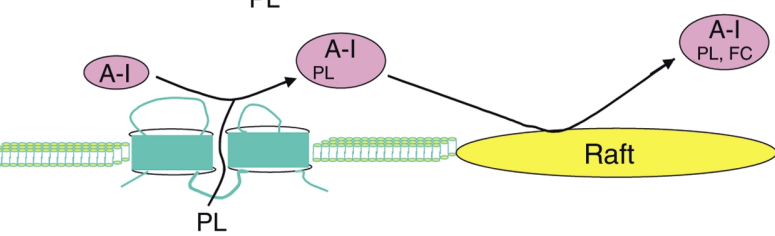

Figure 3

Models for ABCA1-mediated lipid efflux. (a) ABCA1 promotes phospholipid (PL) and cholesterol (FC) efflux from a membrane domain in a single step. (b) ABCA1, located in a border region between liquid and cholesterol-rich liquid-ordered domains (rafts), promotes phospholipid and cholesterol efflux to apoA-I. (c) ABCA1 first promotes phospholipid efflux to apoA-I to form intermediate complexes, which then remove cholesterol from rafts. 
border region between liquid and cholesterol-rich liquid-ordered membrane microdomains. Thus, although phospholipid and cholesterol efflux are normally coupled, this model (Figure $3 \mathrm{~b}$ ) predicts that the two processes might be dissociated when cholesterolenriched rafts are far apart or depleted of cholesterol. Although ABCA1 is localized on the cell surface, it is also found in intracellular organelles, including the Golgi apparatus, early and late endosomes, and lysosomes. Intracellular ABCA1 could be involved in lipid efflux, or it might represent nascent ABCA1 or molecules that have been targeted for degradation. However, energy-dependent trafficking of lipid vesicles between intracellular sites and the cell surface appears to be involved in ABCA1-mediated lipid efflux, as disruption of Golgi membranes by brefeldin (35), and inhibitors of calcium-dependent retroendocytosis (36), inhibit apoA-I-mediated lipid efflux. Moreover, macrophages from Niemann-Pick C1-deficient mice, which are defective in cholesterol release from late endosomes and lysosomes, have a severe reduction in apoA-I-mediated cholesterol but not in phospholipid efflux (37). Similarly, defective cholesterol efflux from late endosomes can result from accumulation of sphingomyelin in Niemann-Pick diseases types A and B, resulting in a defect in cholesterol efflux (38). These studies suggest that cholesterol deposited in late endosomes or lysosomes by modified LDL acts as a preferential source of cholesterol for ABCA1-mediated efflux, perhaps because cholesterol-enriched vesicles are routed to plasma membrane cholesterol-rich rafts that donate cholesterol for ABCA1-mediated efflux.

\section{ABCA1, LXRs, and atherogenesis}

Studies in ABCA1 knockout and ABCA1 transgenic mice. ABCA1 knockout (KO) mice accumulate foam cells in their lungs but do not develop spontaneous atherosclerosis. Furthermore, even when crossed onto apoEor LDL receptor-deficient atherogenic backgrounds, the $A B C A 1$ mutation causes no significant difference in the extent of atherosclerotic lesions, compared with controls (39). However, ABCA1 KO mice have reduced levels of VLDL/LDL cholesterol and apoB in these backgrounds, reminiscent of the reductions in apoB lipoproteins in Tangier disease. Thus, a decrease in the atherogenic stimulus provided by apoB lipoproteins may offset the defect in cholesterol efflux that occurs in ABCA1-deficient macrophages. Consistent with this idea, bone marrow transplantation from ABCA1 KO mice into apoE $\mathrm{KO}$ animals results in an approximately $50 \%$ increase in lesion area $(39,40)$. Although apoA-I and apoE plasma levels are slightly reduced $(39,40)$, overall HDL cholesterol levels are unchanged in these animals. The simplest interpretation of these studies is that defective cholesterol efflux from foam cells can exacerbate atherosclerosis.

Of direct relevance to the idea of upregulating ABCA1 expression as a therapeutic modality are the results of overexpressing ABCA1. Here the effects on plasma lipoproteins and atherogenesis have been less consistent in different models. An initial study with
BAC transgenic mice demonstrated increased macrophage and hepatic ABCA1 expression and cholesterol efflux, but no change in plasma HDL levels (41). The lack of change in plasma HDL may have reflected lower expression levels, since two subsequent studies, using either BAC transgenes containing the $A B C A 1$ gene, or the $A P O E$ promoter-enhancer driving the ABCA1 cDNA, found increased expression of ABCA1 in liver and macrophages, as well as increased HDL levels. ABCA1 transgenic mice in the C57BL/6 background had diminished atherosclerosis, with considerable overlap in the distribution of lesion areas between the groups (42). Moreover, crossing this transgene onto the apoE KO background increased atherosclerosis relative to the parental background. In contrast, ABCA1 transgenic mice produced from a BAC clone have diminished atherosclerosis in the apoE KO background (43). A possible explanation for the differing results is that in the negative study (43), the $A P O E$ promoter was used to drive ABCA1 expression in the apoE KO background. Since apoE could be an important ligand for ABCA1, expression of ABCA1 specifically in cells lacking apoE might limit the ability of ABCA1 to promote cholesterol efflux and to reverse foam cell formation. Further studies using tissue-specific overexpression of ABCA1 in liver or macrophages, and different promoters to drive transgenes, might help to clarify these issues.

Antiatherogenic effects of $L X R / R X R$ targets in macrophages. ApoE and LDLR KO mice treated with small-molecule activators of LXR or RXR show a decrease in atherosclerosis $(16,44)$. Treatment of macrophages with LXR or RXR activator increases expression of ABCA1 mRNA and promotes cholesterol efflux to apoA-I (16). Moreover, apoE KO mice treated with LXR activator show increased expression of ABCA1 and ABCG1 mRNAs in lesions, presumably in macrophages (44). Remarkably, while short-term treatment with LXR activators increases plasma triglycerides and HDL in hamsters, chronic treatment in mice leads to no major changes in plasma lipoprotein levels, suggesting an important but poorly understood adaptive change. These findings suggest that increased macrophage cholesterol efflux, even in the absence of plasma lipoprotein changes, is likely to be antiatherogenic. Alternatively, there may be other LXR target genes expressed in the liver, intestine, or elsewhere, with a beneficial effect on atherogenesis, possibly including ABCG5 or ABCG8.

Human studies. In case-control studies, Schaefer et al. (45) found a two- to threefold increase in risk for atherosclerotic cardiovascular disease in subjects with Tangier disease and obligate $A B C A 1$ heterozygotes, relative to age- and sex-matched controls from the Framingham study. Recently, van Dam et al. (46) studied carotid intima-media thickness in subjects with defined heterozygous mutations of $A B C A 1$. These subjects have isolated low HDL levels, decreased on average by about $43 \%$, but normal VLDL and LDL. In these subjects, the efficiency of apoA-I-mediated cholesterol efflux from individuals' fibroblasts correlates with plasma HDL levels. These authors also found that 
carotid intima-media thickness is increased in subjects with $A B C A 1$ mutations compared with controls. Although these studies are suggestive of increased risk in humans with ABCA1 deficiency, the sample sizes are small. Furthermore, the control subjects (being, on average, 5 years younger than ABCA1-deficient individuals) were not perfectly matched to cases, although the conclusions appeared to hold even after age adjustment (46). Thus, while this correlation is promising, definitive evidence relating ABCA1 deficiency to atherogenesis in humans is still lacking.

\section{Macrophage cholesterol efflux as a strategy for treating atherosclerosis}

In principle, the results of recent mouse atherosclerosis studies represent a conceptual watershed for the development of treatment strategies for atherosclerosis. Together with earlier studies on macrophage-specific apoE expression (47), this work demonstrates the feasibility of upregulating macrophage genes that promote cholesterol efflux as an antiatherogenic treatment, and they indicate that small-molecule activators of LXR or RXR may offer a practical approach to achieving this goal. Although LXR activation is associated with upregulation of SREBP1c target genes (48), resulting in troublesome fatty liver and hypertriglyceridemia, the effects on plasma triglycerides appear to be short-lived (44). However, the issue of fatty liver, which is now thought to be a precursor of more serious liver pathology, remains a stumbling block. The various mechanisms that control ABCA1-mediated lipid efflux might also be therapeutically manipulated. In principle, the most successful strategies could involve macrophage-specific upregulation of cholesterol efflux, or perhaps upregulation of ABCG5 and ABCG8 to promote cholesterol excretion from the body.

\section{Conclusions}

Cellular cholesterol efflux is mediated by both active and passive processes and is regulated on multiple levels. The discovery that Tangier disease is caused by mutations in $A B C A 1$ has provided a molecular key to understanding the mechanisms and regulation of active cellular phospholipid and cholesterol efflux. ABCA1 interacts with lipid-free apolipoproteins, promoting phospholipid and cholesterol efflux from cells and giving rise to HDL particles. Based on analogy with other $\mathrm{ABC}$ transporters, $\mathrm{ABCA1}$ may act as a phospholipid translocase facilitating phospholipid binding to apoA-I. Cholesterol may also be translocated with phospholipid, or it could be derived from membrane rafts in a two-step process. ABCA1 gene expression is upregulated in cholesterol-loaded cells as a result of activation of LXR/RXR-mediated gene transcription. LXR and RXR coordinately induce a battery of genes mediating cellular cholesterol efflux, centripetal cholesterol transport, and cholesterol excretion in bile. Small-molecule activators of LXR/RXR or other stimulators of macrophage or intestinal cholesterol efflux hold great promise as future treatments for atherosclerosis.
1. Williams, K.J., and Tabas, I. 1998. The response-to-retention hypothesis of atherogenesis reinforced. Curr. Opin. Lipidol. 9:471-474.

2. Oram, J.F., Albers, J.J., Cheung, M.C., and Bierman, E.L. 1981. The effects of subfractions of high density lipoprotein on cholesterol efflux from cultured fibroblasts. Regulation of low density lipoprotein receptor activity. J. Biol. Chem. 256:8348-8356.

3. Hara, H., and Yokoyama, S. 1991. Interaction of free apolipoproteins with macrophages. Formation of high density lipoprotein-like lipoproteins and reduction of cellular cholesterol. J. Biol. Chem. 266:3080-3086.

4. Rubin, E.M., Krauss, R.M., Spangler, E.A., Verstuyft, J.G., and Clift, S.M. 1991. Inhibition of early atherogenesis in transgenic mice by human apolipoprotein AI. Nature. 353:265-267.

5. Plump, A.S., Scott, C.J., and Breslow, J.L. 1994. Human apolipoprotein A-I gene expression increases high density lipoprotein and suppresses atherosclerosis in the apolipoprotein E-deficient mouse. Proc. Natl. Acad. Sci. USA. 91:9607-9611.

6. Francis, G.A., Knopp, R.H., and Oram, J.F. 1995. Defective removal of cellular cholesterol and phospholipids by apolipoprotein A-I in Tangier disease. J. Clin. Invest. 96:78-87.

7. Lawn, R.M., Wade, D.P., Couse, T.L., and Wilcox, J.N. 2001. Localization of human ATP-binding cassette transporter 1 (ABC1) in normal and atherosclerotic tissues. Arterioscler. Thromb. Vasc. Biol. 21:378-385.

8. Langmann, T., et al. 1999. Molecular cloning of the human ATP-binding cassette transporter 1 (hABC1): evidence for sterol-dependent regulation in macrophages. Biochem. Biophys. Res. Commun. 257:29-33.

9. Fu, X., et al. 2001. 27-Hydroxycholesterol is an endogenous ligand for liver $\mathrm{X}$ receptor in cholesterol-loaded cells. J. Biol. Chem. 276:38378-38387.

10. Björkhem, I. 2002. Do oxysterols control cholesterol homeostasis? J. Clin. Invest. 110:725-730. doi:10.1172/JCI200216388.

11. Gan, X., et al. 2001. Dual mechanisms of ABCA1 regulation by geranylgeranyl pyrophosphate. J. Biol. Chem. 276:48702-48708.

12. Lin, G., and Bornfeldt, K.E. 2002. Cyclic AMP-specific phosphodiesterase 4 inhibitors promote ABCA1 expression and cholesterol efflux. Biochem. Biophys. Res. Commun. 290:663-669.

13. Panousis, C.G., and Zuckerman, S.H. 2000. Interferon-gamma induces downregulation of Tangier disease gene (ATP-binding-cassette transporter 1) in macrophage-derived foam cells. Arterioscler. Thromb. Vasc. Biol. 20:1565-1571.

14. Chawla, A., et al. 2001. A PPAR gamma-LXR-ABCA1 pathway in macrophages is involved in cholesterol efflux and atherogenesis. Mol. Cell. 7:161-171.

15. Akiyama, T.E., et al. 2002. Conditional disruption of the peroxisome proliferator-activated receptor gamma gene in mice results in lowered expression of ABCA1, ABCG1, and apoE in macrophages and reduced cholesterol efflux. Mol. Cell. Biol. 22:2607-2619.

16. Claudel, T., et al. 2001. Reduction of atherosclerosis in apolipoprotein E knockout mice by activation of the retinoid X receptor. Proc. Natl. Acad. Sci. USA. 98:2610-2615.

17. Oliver, W.R., Jr., et al. 2001. A selective peroxisome proliferator-activated receptor delta agonist promotes reverse cholesterol transport. Proc. Natl. Acad. Sci. USA. 98:5306-5311.

18. Ou, J., et al. 2001. Unsaturated fatty acids inhibit transcription of the sterol regulatory element-binding protein-1c (SREBP-1c) gene by antagonizing ligand-dependent activation of the LXR. Proc. Natl. Acad. Sci. USA. 98:6027-6032.

19. Wang, Y., and Oram, J.F. 2002. Unsaturated fatty acids inhibit cholesterol efflux from macrophages by increasing degradation of ATP-binding cassette transporter A1. J. Biol. Chem. 277:5692-5697.

20. Arakawa, R., and Yokoyama, S. 2002. Helical apolipoproteins stabilize ATP-binding cassette transporter A1 by protecting it from thiol protease-mediated degradation. J. Biol. Chem. 277:22426-22429.

21. Rothblat, G.H., et al. 1999. Cell cholesterol efflux: integration of old and new observations provides new insights. J. Lipid Res. 40:781-796.

22. Acton, S., et al. 1996. Identification of scavenger receptor SR-BI as a high density lipoprotein receptor. Science. 271:518-520.

23. Ji, Y., et al. 1997. Scavenger receptor BI promotes high density lipoprotein-mediated cellular cholesterol efflux. J. Biol. Chem. 272:20982-20985.

24. Gu, X., Kozarsky, K., and Krieger, M. 2000. Scavenger receptor class B, type I-mediated $[3 \mathrm{H}]$ cholesterol efflux to high and low density lipoproteins is dependent on lipoprotein binding to the receptor. J. Biol. Chem. 275:29993-30001.

25. Wang, N., Silver, D.L., Costet, P., and Tall, A.R. 2000. Specific binding of ApoA-I, enhanced cholesterol efflux, and altered plasma membrane morphology in cells expressing ABC1. J. Biol. Chem. 275:33053-33058.

26. Phillips, M.C., Johnson, W.J., and Rothblat, G.H. 1987. Mechanisms and consequences of cellular cholesterol exchange and transfer. Biochim. Biophys. Acta. 906:223-276.

27. Liadaki, K.N., et al. 2000. Binding of high density lipoprotein (HDL) and discoidal reconstituted HDL to the HDL receptor scavenger receptor class B type I. Effect of lipid association and APOA-I mutations on recep- 
tor binding. J. Biol. Chem. 275:21262-21271.

28. Wang, N., Silver, D.L., Thiele, C., and Tall, A.R. 2001. ATP-binding cassette transporter A1 (ABCA1) functions as a cholesterol efflux regulatory protein. J. Biol. Chem. 276:23742-23747.

29. Fielding, C.J., and Fielding, P.E. 2001. Cellular cholesterol efflux Biochim. Biophys. Acta. 1533:175-189.

30. Bungert, S., Molday, L.L., and Molday, R.S. 2001. Membrane topology of the ATP binding cassette transporter $\mathrm{ABCR}$ and its relationship to $\mathrm{ABC} 1$ and related $\mathrm{ABCA}$ transporters: identification of N-linked glycosylation sites. J. Biol. Chem. 276:23539-23546.

31. Remaley, A.T., et al. 2001. Apolipoprotein specificity for lipid efflux by the human ABCAI transporter. Biochem. Biophys. Res. Commun. 280:818-823.

32. Oram, J.F., Lawn, R.M., Garvin, M.R., and Wade, D.P. 2000. ABCA1 is the cAMP-inducible apolipoprotein receptor that mediates cholesterol secretion from macrophages. J. Biol. Chem. 275:34508-34511.

33. Fitzgerald, M.L., et al. 2002. Naturally occurring mutations in the largest extracellular loops of ABCA1 can disrupt its direct interaction with apolipoprotein A-I. J. Biol. Chem. 277:33178-33187.

34. Chambenoit, O., et al. 2001. Specific docking of apolipoprotein A-I at the cell surface requires a functional ABCA1 transporter. J. Biol. Chem. 276:9955-9960.

35. Neufeld, E.B., et al. 2001. Cellular localization and trafficking of the human ABCA1 transporter. J. Biol. Chem. 276:27584-27590.

36. Takahashi, Y., and Smith, J.D. 1999. Cholesterol efflux to apolipoprotein AI involves endocytosis and resecretion in a calcium-dependent pathway. Proc. Natl. Acad. Sci. USA. 96:11358-11363.

37. Chen, W., et al. 2001. Preferential ATP-binding cassette transporter A1-mediated cholesterol efflux from late endosomes/lysosomes. J. Biol. Chem. 276:43564-43569.

38. Leventhal, A.R., Chen, W., Tall, A.R., and Tabas, I. 2001. Acid sphingomyelinase-deficient macrophages have defective cholesterol trafficking and efflux. J. Biol. Chem. 276:44976-44983.
39. Aiello, R.J., et al. 2002. Increased atherosclerosis in hyperlipidemic mice with inactivation of ABCA1 in macrophages. Arterioscler. Thromb. Vasc. Biol. 22:630-637.

40. van Eck, M., et al. 2002. Leukocyte ABCA1 controls susceptibility to atherosclerosis and macrophage recruitment into tissues. Proc. Natl. Acad. Sci. USA. 99:6298-6303.

41. Cavelier, L.B., et al. 2001. Regulation and activity of the human ABCA1 gene in transgenic mice. J. Biol. Chem. 276:18046-18051.

42. Joyce, C.W., et al. 2002. The ATP binding cassette transporter A1 (ABCA1) modulates the development of aortic atherosclerosis in C57BL/6 and apoE-knockout mice. Proc. Natl. Acad. Sci. USA 99:407-412.

43. Singaraja, R.R., et al. 2002. Increased ABCA1 activity protects against atherosclerosis. J. Clin. Invest. 110:35-42. doi:10.1172/JCI200215748.

44. Joseph, S.B., et al. 2002. Synthetic LXR ligand inhibits the developmen of atherosclerosis in mice. Proc. Natl. Acad. Sci. USA. 99:7604-7609.

45. Schaefer, E.J., Zech, L.A., Schwartz, D.E., and Brewer, H.B., Jr. 1980 Coronary heart disease prevalence and other clinical features in familial high-density lipoprotein deficiency (Tangier disease). Ann. Intern. Med. 93:261-266.

46. van Dam, M.J., et al. 2002. Association between increased arterial-wall thickness and impairment in ABCA1-driven cholesterol efflux: an observational study. Lancet. 359:37-42.

47. Bellosta, S., et al. 1995. Macrophage-specific expression of human apolipoprotein E reduces atherosclerosis in hypercholesterolemic apolipoprotein E-null mice. J. Clin. Invest. 96:2170-2179.

48. Repa, J.J., et al. 2000. Regulation of mouse sterol regulatory elementbinding protein-1c gene (SREBP-1c) by oxysterol receptors, LXRalpha and LXRbeta. Genes Dev. 14:2819-2830.

49. Fitzgerald, M.L., et al. 2001. ATP-binding cassette transporter A1 contains an NH2-terminal signal anchor sequence that translocates the protein's first hydrophilic domain to the exoplasmic space. J. Biol. Chem. 276:15137-15145. 